\title{
Synchronization of Different Fractional Order Time-Delay Chaotic Systems Using Active Control
}

\author{
Jianeng Tang \\ College of Engineering, Huaqiao University, Quanzhou, Fujian 362021, China \\ Correspondence should be addressed to Jianeng Tang; tangjianeng@sina.com
}

Received 7 November 2013; Accepted 16 January 2014; Published 23 February 2014

Academic Editor: Santanu Saha Ray

Copyright (C) 2014 Jianeng Tang. This is an open access article distributed under the Creative Commons Attribution License, which permits unrestricted use, distribution, and reproduction in any medium, provided the original work is properly cited.

Chaos synchronization of different fractional order time-delay chaotic systems is considered. Based on the Laplace transform theory, the conditions for achieving synchronization of different fractional order time-delay chaotic systems are analyzed by use of active control technique. Then numerical simulations are provided to verify the effectiveness and feasibility of the developed method. At last, effects of the fraction order and the time delay on synchronization are further researched.

\section{Introduction}

Since the pioneering work of Pecora and Carroll [1], there has been a significant interest in developing powerful techniques for chaos synchronization in the past 20 years. Some different regimes of chaos synchronization have been studied. They contain partial synchronization [2], complete synchronization $[3,4]$, phase synchronization $[5,6]$, generalized synchronization [7, 8], projective synchronization [9], and lag synchronization [10]. Of course, Robust synchronization is an important aspect of chaos synchronization. Based on the Lyapunov stability theory and LMI technique, a new sufficient criterion is established for chaos robust synchronization [11]. By use of the sliding mode control technique, a robust control scheme is established even when the parameter uncertainty and external perturbation are present [12]. A robust antisynchronization scheme is proposed according to multiple-kernel least squares support vector machine modeling for two uncertain chaotic systems [13]. Many potential applications of chaos synchronization, especially in secure communications of analog and digital signals and for developing safe and reliable cryptographic systems, chemical oscillators, and electronic systems, have been researched.

It is that delayed differential equations have been largely investigated in $[14,15]$ and references cited therein. Time delays and time-varying delays are recently introduced to chaotic systems; for example, see [16-19]. However, most of these publications are for integer-order or typical differential equations. Although fractional calculus is a 300 -year-old mathematical topic, for many years it was not used in physics and engineering. During the last 10 years or so, fractional calculus starts to become focus of attention of physicists and engineers $[20,21]$. There are many known systems to display fractional-order dynamics, such as dielectric polarization, electromagnetic waves, and quantum evolution of complex systems. Thus it has been a new trend, that is, the fractional power of the control system dynamics [22-35].

According to the Poincare-Bendixon theorem [22], an integer order chaotic nonlinear system must have a minimum order of 3 for chaos to appear. However, it is not the case in the fractional order nonlinear systems. Some examples in the respect are $\mathrm{Wu}$ et al. [23] (unified system), $\mathrm{Lu}$ and Chen [24] (Chen system), and Lu [25] (Ikeda system). By using Lambert function, the analytical stability bound is obtained for delayed second-order systems with repeatable poles and the bound is obtained delayed linear time-invariant fractional-order dynamic systems [26, 27]. Based on the Laplace transform theory, synchronization scheme to chaotic fractional-order Chen systems is derived in [28]; Deng et al. [29] introduced a characteristic equation for the ndimensional linear fractional differential system with multiple time delays. In line with the stability theorem of linear fractional systems, a necessary condition is given to check 
the chaos synchronization of fractional systems with incommensurate order [30]. In [31], Shao et al. proposed a method to achieve projective synchronization of the fractional order chaotic Rossler system. In [32], an analytical justification was proposed for phase synchronization of fractional differential equations. A stability test procedure was proposed for linear nonhomogeneous fractional order systems with a pure time delay [33]. Taghvafard and Erjaee [34] studied the phase and antiphase synchronization between two identical and nonidentical fractional order chaotic systems using techniques from active control theory. The effect of delay on the chaotic behaviour has been investigated for the first time in the literature [35].

However, there are few results about chaos synchronization of the fractional order time-delay chaotic systems. In our work, we give an improved version of Adams-BashforthMoulton method. By use of active control technique, the conditions for achieving synchronization of different fractional order time-delay chaotic systems are analyzed based on the Laplace transform theory. Then effects of the fraction order and the time delay on synchronization are further analyzed.

The organization of this paper is as follows. In Section 2, some definitions and systems are given and then an improved version of Adams-Bashforth-Moulton method is introduced. In the following three sections, we synchronize the following fractional systems using active control method: (i) Liu with Liu, (ii) Lü with Chen, and (iii) Lorenz with Chen. The effectiveness of our work is verified through numerical simulations. In Section 6, effects of the fraction order and the time delay on synchronization are further researched. Finally concluding remark is given.

\section{Definitions and Systems}

2.1. Fractional Calculus. There are several definitions of a fractional-order differential system. In the following, we introduce the most common one of them:

$$
D_{*}^{a} x(t)=\frac{d^{\alpha} x}{d t^{\alpha}}=J^{m-\alpha} x^{(m)}(t)
$$

where $m=[\alpha]$; that is, $m$ is the first integer which is not less than $\alpha(0<\alpha<1), x^{(m)}$ is the $m$-order derivative in the usual sense, and $J^{\beta}(\beta>0)$ is the $\beta$-order Reimann-Liouville integral operator with expression:

$$
J^{\beta} y(t)=\frac{1}{\Gamma(\beta)} \int_{0}^{t}(t-\tau)^{\beta-1} y(\tau) d_{\tau}
$$

Here $\Gamma$ stands for Gamma function, and the operator $D_{*}^{\alpha}$ is generally called “ $\alpha$-order Caputo differential operator" [33].

2.2. System Description. The fractional order Lorenz system has recently been studied in many references $[36,37]$. Here we

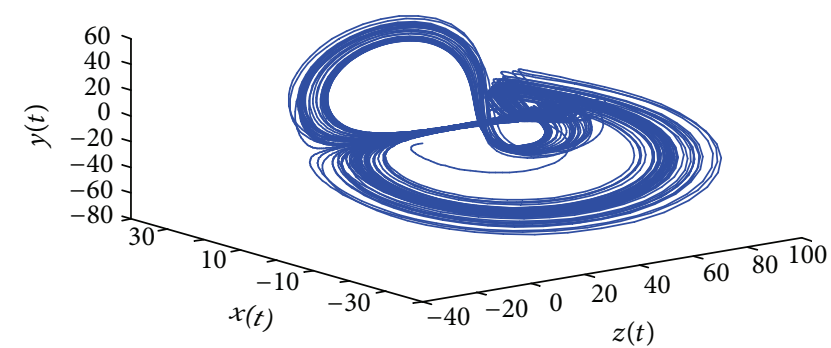

FIGURE 1: The fractional-order time-delay Lorenz chaotic attractors with $\sigma=10, \mu=8 / 3, r=28, \alpha=0.95$, and $\tau=0.4$.

give a fractional order time-delay Lorenz system. The system is described by

$$
\begin{gathered}
\frac{d^{\alpha} x}{d t^{\alpha}}=\sigma(y-x), \\
\frac{d^{\alpha} y}{d t^{\alpha}}=r x-y-x z, \\
\frac{d^{\alpha} z}{d t^{\alpha}}=x y-\mu z(t-\tau),
\end{gathered}
$$

where $\sigma=10$ is the Prandtl number, $r=28$ is the Rayleigh number over the critical Rayleigh number, $\mu=8 / 3$ gives the size of the region approximated by the system, and $\tau>0$ is time delay. System (3) displays a chaotic attractor, as shown in Figure 1.

In 1999, Chen and Ueta [38] introduced a new chaotic system, which is similar but not topologically equivalent to the Lorenz system. Without changing the model structure of the system, we consider the fractional order time-delay Chen system in the following form:

$$
\begin{gathered}
\frac{d^{\alpha} x}{d t^{\alpha}}=a(y-x), \\
\frac{d^{\alpha} y}{d t^{\alpha}}=(c-a) x-x z+c y, \\
\frac{d^{\alpha} z}{d t^{\alpha}}=x y-b z(t-\tau),
\end{gathered}
$$

where $a, b$, and $c$ are real parameters. When $a=35, b=3$, and $c=28$, system (4) displays a chaotic attractor through the suitable selection of time delay, as shown in Figure 2. The fractional order time-delay Lü system is given by

$$
\begin{gathered}
\frac{d^{\alpha} x}{d t^{\alpha}}=a_{1}(y-x), \\
\frac{d^{\alpha} y}{d t^{\alpha}}=c_{1} x-x z, \\
\frac{d^{\alpha} z}{d t^{\alpha}}=x y-b_{1} z(t-\tau),
\end{gathered}
$$

where $a_{1}=35, b_{1}=3$, and $c_{1}=28$. As shown in Figure 3, system (5) displays a chaotic attractor through the suitable selection of time delay. 


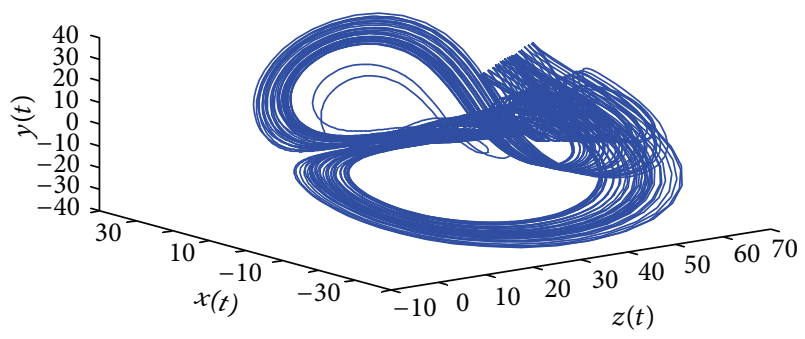

FIgURE 2: The fractional-order time-delay Chen chaotic attractors with $a=35, b=3, c=28, \alpha=0.98$, and $\tau=0.2$.

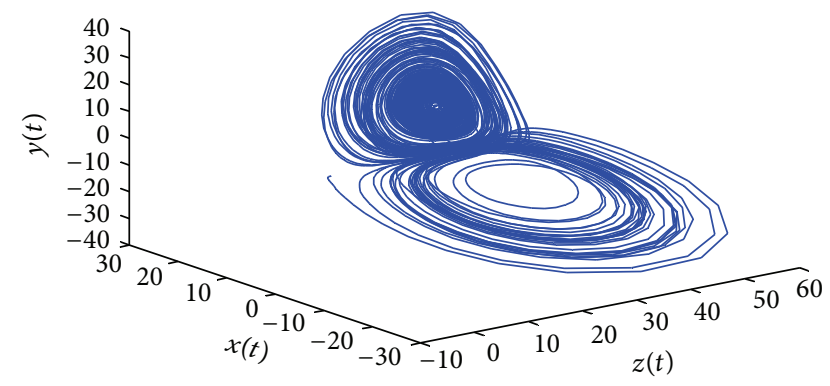

FIGURE 3: The fractional-order time-delay Lü chaotic attractors with $a_{1}=35, b_{1}=3, c_{1}=28, \alpha=0.78$, and $\tau=1$.

In 2009, Liu et al. proposed a novel three-dimensional autonomous chaos system (called Liu system) [39]. Daftardar-Gejji and Bhalekar [40] studied a fractional version of the chaotic system. Here we investigate a fractional order time-delay Liu system as follows:

$$
\begin{gathered}
\frac{d^{\alpha} x}{d t^{\alpha}}=-a_{2} x-e y^{2}, \\
\frac{d^{\alpha} y}{d t^{\alpha}}=b_{2} x-k x z, \\
\frac{d^{\alpha} z}{d t^{\alpha}}=m x y-c_{2} z(t-\tau),
\end{gathered}
$$

where $a_{2}=1, b_{2}=2.5, c_{2}=5, e=1, k=4$, and $m=4$. The system can be in the chaotic state as shown in Figure 4.

2.3. Numerical Method. According to, [41-43] the predictorcorrector scheme for system (4) is derived. Here we give an improved version of Adams-Bashforth-Moulton method [43]. Based on the fractional order time-delay Chen system, let us illustrate this scheme.

The following differential equation:

$$
\begin{gathered}
\frac{d^{\alpha} x}{d t^{\alpha}}=f(t, x), \quad 0 \leq t \leq T, \\
x^{(k)}(0)=x_{0}^{(k)}, \quad k=0,1,2, \ldots, n-1,
\end{gathered}
$$

is equivalent to the Volterra integral equation [42]

$$
x(t)=\sum_{k=0}^{n-1} x_{0}^{(k)} \frac{t^{k}}{k !}+\frac{1}{\Gamma(\alpha)} \int_{0}^{t} \frac{f(\tau 1, x)}{(t-\tau)^{1-\alpha}} d \tau 1 .
$$

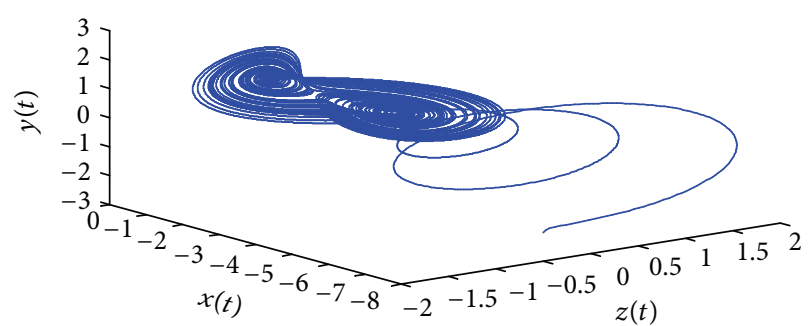

FIGURE 4: The fractional-order time-delay Liu chaotic attractors with $a_{2}=1, b_{2}=2.5, c_{2}=5, e=1, k=4, m=5, \alpha=0.985$, and $\tau=0.002$.

Let $h=T / N, t_{n}=n h(n=0,1,2, \ldots, N)$, and $n 1=\tau / h$. Then (8) can be discretized as follows:

$$
\begin{aligned}
x_{h}\left(t_{n+1}\right)= & \sum_{k=0}^{n-1} x_{0}^{(k)} \frac{t_{n}^{k+1}}{k !}+\frac{h^{\alpha}}{\Gamma(\alpha+2)} f\left(t_{n+1}, x_{h}^{\rho}\left(t_{n+1}\right)\right) \\
& +\frac{h^{\alpha}}{\Gamma(\alpha+2)} \sum a_{j, n+1} f\left(t_{j}, x_{h}\left(t_{j}\right)\right),
\end{aligned}
$$

where

$$
\begin{gathered}
a_{j, n+1}= \begin{cases}n^{\alpha+1}-(n-\alpha)(n+1)^{\alpha+1}, & j=0 \\
(n-j+2)^{\alpha+1}+(n-j)^{\alpha+1} & \\
-2(n-j+1)^{\alpha+1}, & 1 \leq j \leq n \\
1, & j=n+1,\end{cases} \\
x_{h}^{\rho}\left(t_{n+1}\right)=\sum_{k=0}^{n-1} x_{0}^{(k)} \frac{t_{n}^{k+1}}{k !}+\frac{1}{\Gamma(\alpha)} \sum_{j=0}^{n} b_{j, n+1} f\left(t_{j}, x_{h}\left(t_{j}\right)\right), \\
b_{j, n+1}=\frac{h^{\alpha}}{\alpha}\left((n-j+1)^{\alpha}-(n-j)^{\alpha}\right) .
\end{gathered}
$$

The error estimate is $e=\operatorname{Max}\left|x\left(t_{j}\right)-x_{h}\left(t_{j}\right)\right|=O\left(h^{\rho}\right)(j=$ $0,1, \ldots, N)$ where $\rho=\operatorname{Min}(2,1+\alpha)$.

Applying the above method, (4) can be discretized as follows:

$$
\begin{aligned}
x_{n+1}= & x_{0}+\frac{h^{\alpha}}{\Gamma(\alpha+2)} a\left(y_{n+1}^{\rho}-x_{n+1}^{\rho}\right) \\
& +\frac{h^{\alpha}}{\Gamma(\alpha+2)} \sum_{j=0}^{n} \gamma_{1, j, n+1} a\left(y_{j}-x_{j}\right), \\
y_{n+1}= & y_{0}+\frac{h^{\alpha}}{\Gamma(\alpha+2)}\left[(c-a) x_{n+1}^{\rho}-x_{n+1}^{\rho} z_{n+1}^{\rho}+c y_{n+1}^{\rho}\right] \\
& +\frac{h^{\alpha}}{\Gamma(\alpha+2)} \sum_{j=0}^{n} \gamma_{2, j, n+1}\left[(c-a) x_{j}-x_{j} z_{j}+c y_{j}\right] \\
z_{n+1}= & z_{0}+\frac{h^{\alpha}}{\Gamma(\alpha+2)}\left(x_{n+1}^{\rho} y_{n+1}^{\rho}-b z_{n+1-n 1}^{\rho}\right) \\
& +\frac{h^{\alpha}}{\Gamma(\alpha+2)} \sum_{j=0}^{n} \gamma_{3, j, n+1}\left(x_{j} y_{j}-b z_{j-n 1}\right),
\end{aligned}
$$


where

$$
\begin{gathered}
x_{n+1}^{\rho}=x_{0}+\frac{1}{\Gamma(\alpha+2)} \sum_{j=0}^{n} \omega_{1, j, n+1} a\left(y_{j}-x_{j}\right), \\
y_{n+1}^{\rho}=y_{0}+\frac{1}{\Gamma(\alpha+2)} \sum_{j=0}^{n} \omega_{2, j, n+1}\left[(c-a) x_{j}-x_{j} z_{j}+c y_{j}\right], \\
z_{n+1}^{\rho}=z_{0}+\frac{1}{\Gamma(\alpha+2)} \sum_{j=0}^{n} \omega_{3, j, n+1}\left(x_{j} y_{j}-b z_{j-n 1}\right), \\
\gamma_{i, j, n+1}=\left\{\begin{array}{cc}
n^{\alpha+1}-(n-\alpha)(n+1)^{\alpha+1}, & j=0 \\
(n-j+2)^{\alpha+1}+(n-j)^{\alpha+1} & \\
1, & j \leq j+j)^{\alpha+1}, \\
\omega_{i, j, n+1}=\frac{h^{\alpha}}{\alpha}\left((n-j+1)^{\alpha}-(n-j)^{\alpha}\right), \\
0 \leq j \leq n, & i=1,2,3 .
\end{array}\right.
\end{gathered}
$$

\section{Chaos Synchronization between Fractional Order Time-Delay Liu and Chen System}

In this section we study the synchronization between Liu and Chen systems. Assuming that the Liu system drives the Chen system, we define the drive (master) and response (slave) systems as follows:

$$
\begin{gathered}
\frac{d^{\alpha} x_{1}}{d t^{\alpha}}=-a_{1} x_{1}-e y_{1}^{2}, \\
\frac{d^{\alpha} y_{1}}{d t^{\alpha}}=b_{1} x_{1}-k x_{1} z_{1}, \\
\frac{d^{\alpha} z_{1}}{d t^{\alpha}}=m x_{1} y_{1}-c_{1} z_{1}(t-\tau), \\
\frac{d^{\alpha} x_{2}}{d t^{\alpha}}=a_{2}\left(y_{2}-x_{2}\right)+u_{1}(t), \\
\frac{d^{\alpha} y_{2}}{d t^{\alpha}}=\left(c_{2}-a_{2}\right) x_{2}-x_{2} z_{2}+c_{2} y_{2}+u_{2}(t), \\
\frac{d^{\alpha} z_{2}}{d t^{\alpha}}=x_{2} y_{2}-b_{2} z_{2}(t-\tau)+u_{3}(t) .
\end{gathered}
$$

The unknown terms $u_{1}, u_{2}$, and $u_{3}$ in (14) are active control functions to be determined. Define the error functions as

$$
e_{1}=x_{2}-x_{1}, \quad e_{2}=y_{2}-y_{1}, \quad e_{3}=z_{2}-z_{1} .
$$

Equation (15) together with (13) and (14) yields the error system

$$
\frac{d^{\alpha} e_{1}}{d t^{\alpha}}=a_{2}\left(y_{2}-x_{2}\right)+u_{1}(t)+a_{1} x_{1}+e y_{1}^{2},
$$

$$
\begin{aligned}
& \frac{d^{\alpha} e_{2}}{d t^{\alpha}}=\left(c_{2}-a_{2}\right) x_{2}-x_{2} z_{2}+c_{2} y_{2}+u_{2}(t)-b_{1} x_{1}+k x_{1} z_{1}, \\
& \frac{d^{\alpha} e_{3}}{d t^{\alpha}}=x_{2} y_{2}-b_{2} z_{2}(t-\tau)+u_{3}(t)-m x_{1} y_{1}+c_{1} z_{1}(t-\tau) .
\end{aligned}
$$

By using Matignon's theorem [44], so we here define active control function $u_{i}(i=1,2,3)$ as

$$
\begin{aligned}
u_{1}(t)= & -3 e_{1}-2 e_{1}(t-\tau)-4 e_{2} \\
& -3 e_{2}(t-\tau)-a_{2}\left(y_{2}-x_{2}\right)-a_{1} x_{1}-e y_{1}^{2}, \\
u_{2}(t)= & -6 e_{2}-5 e_{2}(t-\tau)-\left(c_{2}-a_{2}\right) x_{2} \\
& +x_{2} z_{2}-c_{2} y_{2}+b_{1} x_{1}-k x_{1} z_{1}, \\
u_{3}(t)= & -2 e_{1}-e_{1}(t-\tau)-8 e_{2}-3 e_{2}(t-\tau) \\
& -10 e_{3}-7 e_{3}(t-\tau)-x_{2} y_{2}+b_{2} z_{2}(t-\tau) \\
& +m x_{1} y_{1}-c_{1} z_{1}(t-\tau) .
\end{aligned}
$$

Substituting (17) into (16), we achieve

$$
\begin{aligned}
\frac{d^{\alpha} e_{1}}{d t^{\alpha}}=- & -3 e_{1}-2 e_{1}(t-\tau)-4 e_{2}-3 e_{2}(t-\tau), \\
& \frac{d^{\alpha} e_{2}}{d t^{\alpha}}=-6 e_{2}-5 e_{2}(t-\tau), \\
\frac{d^{\alpha} e_{3}}{d t^{\alpha}}= & -2 e_{1}-e_{1}(t-\tau)-8 e_{2} \\
& -3 e_{2}(t-\tau)-10 e_{3}-7 e_{3}(t-\tau) .
\end{aligned}
$$

Then we start to prove that the error system (18) is asymptotically stable; that is, the system (13) and the system (14) with the active control law (17) are synchronized.

Taking the Laplace transformation in two sides of (18), letting $E_{i}(s)=L\left(e_{i}(t)\right)(i=1,2,3)$, and utilizing $L\left(d^{\alpha} e_{i} /\right.$ $\left.d t^{\alpha}\right)=s^{\alpha} E_{i}(s)-s^{\alpha-1} e_{i}(0)$ (see [21]), we obtain

$$
\begin{aligned}
& s^{\alpha} E_{1}(s)-s^{\alpha-1} e_{1}(0) \\
&=-3 E_{1}(s)-2 E_{1}(s) e^{-s \tau}-4 E_{2}(s)-3 E_{2}(s) e^{-s \tau}, \\
& s^{\alpha} E_{2}(s)-s^{\alpha-1} e_{2}(0)=-6 E_{2}(s)-5 E_{2}(s) e^{-s \tau}, \\
& s^{\alpha} E_{3}(s)-s^{\alpha-1} e_{3}(0) \\
&=-2 E_{1}(s)-E_{1}(s) e^{-s \tau}-8 E_{2}(s)-3 E_{2}(s) e^{-s \tau} \\
& \quad-10 E_{3}(s)-7 E_{3}(s) e^{-s \tau} .
\end{aligned}
$$


Equation (19) can be rewritten as follows:

$$
\begin{aligned}
& E_{1}(s)=\frac{s^{\alpha-1} e_{1}(0)+\left(-4-3 e^{-s \tau}\right) E_{2}(s)}{s^{\alpha}+3+2 e^{-s \tau}}, \\
& E_{2}(s)=\frac{s^{\alpha-1} e_{2}(0)}{s^{\alpha}+6+5 e^{-s \tau}}, \\
& E_{3}(s)=\frac{s^{\alpha-1} e_{3}(0)+\left(-2-e^{-s \tau}\right) E_{1}(s)+\left(-8-3 e^{-s \tau}\right) E_{2}(s)}{s^{\alpha}+10+7 e^{-s \tau}} .
\end{aligned}
$$

By use of the final-value theorem of the Laplace transformation, we have

$$
\lim _{t \rightarrow+\infty} e_{2}(t)=\lim _{s \rightarrow 0^{+}} s E_{2}(s)=\frac{s^{\alpha} e_{2}(0)}{s^{\alpha}+6+5 e^{-s \tau}}=0 .
$$

At the same time, from (20), we can get

$$
\begin{aligned}
\lim _{t \rightarrow+\infty} e_{1}(t) & \\
= & \lim _{s \rightarrow 0^{+}} s E_{1}(s) \\
& =\frac{s^{\alpha} e_{1}(0)+s\left(-4-3 e^{-s \tau}\right) E_{2}(s)}{s^{\alpha}+3+2 e^{-s \tau}}=0, \\
\lim _{t \rightarrow+\infty} e_{3}(t) & \\
= & \lim _{s \rightarrow 0^{+}} s E_{3}(s) \\
= & \frac{s^{\alpha} e_{3}(0)+s\left(-2-e^{-s \tau}\right) E_{1}(s)+s\left(-8-3 e^{-s \tau}\right) E_{2}(s)}{s^{\alpha}+10+7 e^{-s \tau}}
\end{aligned}
$$$$
=0 .
$$

The above analysis implies that the fractional-order drive system (13) and the fractional-order response system (14) with the active control law (17) are synchronized.

3.1. Simulation Research. Here, let $a_{1}=1, b_{1}=2.5, c_{1}=5$, $e=1, k=4, m=5$ in the Liu system and parameters of the Chen system are taken as $a_{2}=35, b_{2}=3, c_{2}=28$. The fractional order $\alpha$ is taken to be 0.985 and assume $\tau=$ 0.002 . The initial conditions for drive and response system are $x_{1}(0)=-8, y_{1}(0)=-2, z_{1}(0)=-0.5$ and $x_{2}(0)=0.5$, $y_{2}(0)=1, z_{2}(0)=10$, respectively. Thus initial conditions for the error system are $e_{1}(0)=8.5, e_{2}(0)=3, e_{3}(0)=10.5$. Figures $5(\mathrm{a})-5(\mathrm{c})$ shows the synchronization between Chen and Liu system. The errors $e_{i}(t)$ for the drive and response system are shown in Figure 5(d).

\section{Chaos Synchronization between Fractional Order Time-Delay Lü and Chen System}

In this section we consider Lü system as the drive systems

$$
\begin{gathered}
\frac{d^{\alpha} x_{1}}{d t^{\alpha}}=a_{1}\left(y_{1}-x_{1}\right), \\
\frac{d^{\alpha} y_{1}}{d t^{\alpha}}=c_{1} x_{1}-x_{1} z_{1}, \\
\frac{d^{\alpha} z_{1}}{d t^{\alpha}}=x_{1} y_{1}-b_{1} z_{1}(t-\tau),
\end{gathered}
$$

and the Chen system as the response system

$$
\begin{gathered}
\frac{d^{\alpha} x_{2}}{d t^{\alpha}}=a_{2}\left(y_{2}-x_{2}\right)+u_{1}(t), \\
\frac{d^{\alpha} y_{2}}{d t^{\alpha}}=\left(c_{2}-a_{2}\right) x_{2}-x_{2} z_{2}+c_{2} y_{2}+u_{2}(t), \\
\frac{d^{\alpha} z_{2}}{d t^{\alpha}}=x_{2} y_{2}-b_{2} z_{2}(t-\tau)+u_{3}(t) .
\end{gathered}
$$

The unknown terms $u_{1}, u_{2}, u_{3}$ in (24) are active control functions to be determined. Define the error functions as

$$
e_{1}=x_{2}-x_{1}, \quad e_{2}=y_{2}-y_{1}, \quad e_{3}=z_{2}-z_{1}
$$

Equation (25) together with (23) and (24) yields the error system

$$
\begin{gathered}
\frac{d^{\alpha} e_{1}}{d t^{\alpha}}=a_{2}\left(y_{2}-x_{2}\right)+u_{1}(t)-a_{1}\left(y_{1}-x_{1}\right), \\
\frac{d^{\alpha} e_{2}}{d t^{\alpha}}=\left(c_{2}-a_{2}\right) x_{2}-x_{2} z_{2}+c_{2} y_{2}+u_{2}(t)-c_{1} y_{1}+x_{1} z_{1}, \\
\frac{d^{\alpha} e_{3}}{d t^{\alpha}}=x_{2} y_{2}-b_{2} z_{2}(t-\tau)+u_{3}(t)-x_{1} y_{1}+b_{1} z_{1}(t-\tau) .
\end{gathered}
$$

By using of the Matignon's theorem [44], so we here define active control function $u_{i}(i=1,2,3)$ as

$$
\begin{aligned}
u_{1}(t)= & -4 e_{1}-3 e_{1}(t-\tau)-a_{2}\left(y_{2}-x_{2}\right) \\
& +a_{1}\left(y_{1}-x_{1}\right), \\
u_{2}(t)= & -6 e_{1}-3 e_{1}(t-\tau)-7.5 e_{2}-5.3 e_{2}(t-\tau) \\
& -3 e_{3}-e_{3}(t-\tau)-\left(c_{2}-a_{2}\right) x_{2}+x_{2} z_{2} \\
& -c_{2} y_{2}+c_{1} y_{1}-x_{1} z_{1}, \\
u_{3}(t)= & -8 e_{1}-4 e_{1}(t-\tau)-9 e_{3}-2 e_{3}(t-\tau) \\
& -x_{2} y_{2}+b_{2} z_{2}(t-\tau)+x_{1} y_{1}-b_{1} z_{1}(t-\tau) .
\end{aligned}
$$



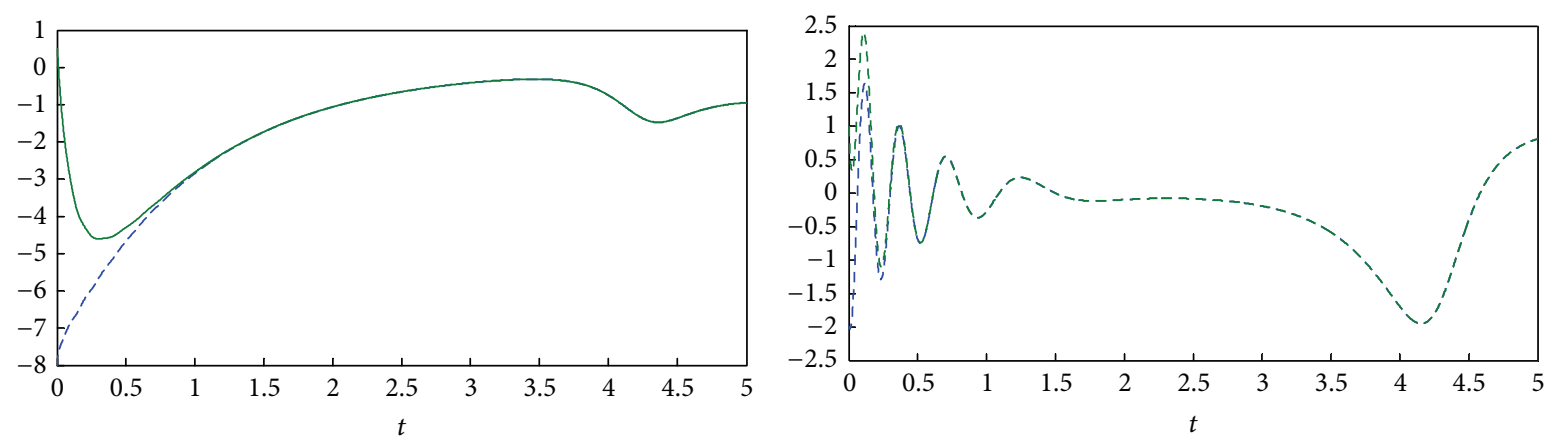

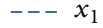

$-x_{2}$

(a)

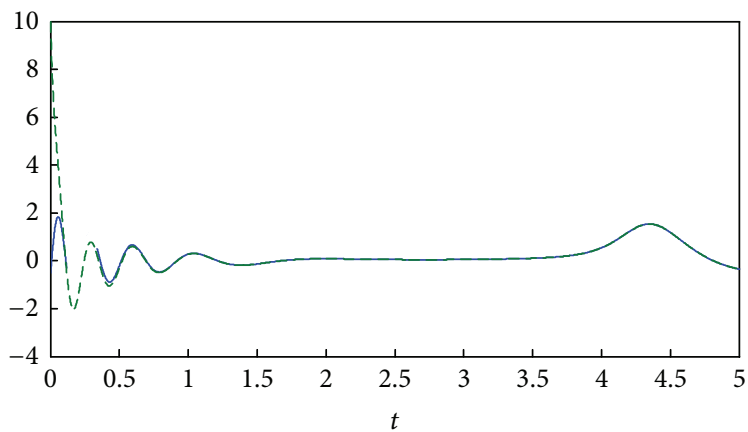

$-z_{1}$ $---y_{1}$

$---y_{2}$

(b)

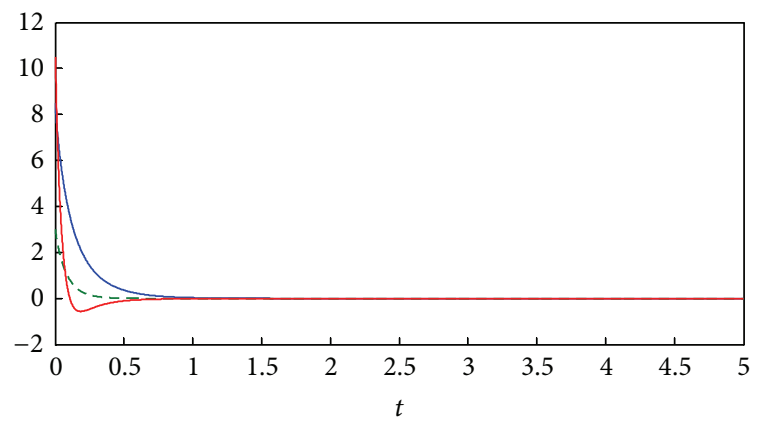

$\begin{aligned}- & e_{1} \\ --- & e_{2}\end{aligned}$

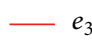

(d)

Figure 5: Chaos synchronization between (13) and (14). (a) Signals $x_{1}, x_{2}$. (b) Signals $y_{1}, y_{2}$. (c) Signals $z_{1}, z_{2}$. (d) Error system.

Substituting (27) into (26), we achieve

$$
\begin{gathered}
\frac{d^{\alpha} e_{1}}{d t^{\alpha}}=-4 e_{1}-3 e_{1}(t-\tau), \\
\frac{d^{\alpha} e_{2}}{d t^{\alpha}}=-6 e_{1}-3 e_{1}(t-\tau)-7.5 e_{2} \\
-5.3 e_{2}(t-\tau)-3 e_{3}-e_{3}(t-\tau), \\
\frac{d^{\alpha} e_{3}}{d t^{\alpha}}=-8 e_{1}-4 e_{1}(t-\tau)-9 e_{3}-2 e_{3}(t-\tau) .
\end{gathered}
$$

Then we start to prove that the error system (28) is asymptotically stable, that is, the system (23) and the system (24) with the active control law (27) are synchronized.

Taking the Laplace transformation in two sides of (28), letting $E_{i}(s)=L\left(e_{i}(t)\right)(i=1,2,3)$, and utilizing $L\left(d^{\alpha} e_{i} /\right.$ $\left.d t^{\alpha}\right)=s^{\alpha} E_{i}(s)-s^{\alpha-1} e_{i}(0)$ (see [18]), we obtain

$$
\begin{aligned}
& s^{\alpha} E_{1}(s)-s^{\alpha-1} e_{1}(0) \\
&=-4 E_{1}(s)-3 E_{1}(s) e^{-s \tau}, \\
& s^{\alpha} E_{2}(s)-s^{\alpha-1} e_{2}(0)
\end{aligned}
$$

$$
\begin{aligned}
= & -6 E_{1}(s)-3 E_{1}(s) e^{-s \tau}-7.5 E_{2}(s) \\
& -5.3 E_{2}(s) e^{-s \tau}-3 E_{3}(s)-E_{3}(s) e^{-s \tau}, \\
s^{\alpha} E_{3}(s) & -s^{\alpha-1} e_{3}(0) \\
= & -8 E_{1}(s)-4 E_{1}(s) e^{-s \tau} \\
& -9 E_{3}(s)-2 E_{3}(s) e^{-s \tau} .
\end{aligned}
$$

Equation (29) can be rewritten as follows:

$$
\begin{aligned}
& E_{1}(s)=\frac{s^{\alpha-1} e_{1}(0)}{s^{\alpha}+4+3 e^{-s \tau}}, \\
& E_{2}(s)=\frac{s^{\alpha-1} e_{2}(0)+\left(-6-3 e^{-s \tau}\right) E_{1}(s)+\left(-3-e^{-s \tau}\right) E_{3}(s)}{s^{\alpha}+7.5+5.3 e^{-s \tau}}, \\
& E_{3}(s)=\frac{s^{\alpha-1} e_{3}(0)+\left(-8-4 e^{-s \tau}\right) E_{1}(s)}{s^{\alpha}+9+2 e^{-s \tau}} .
\end{aligned}
$$

By use of the final-value theorem of the Laplace transformation, we have

$$
\lim _{t \rightarrow+\infty} e_{1}(t)=\lim _{s \rightarrow 0^{+}} s E_{1}(s)=\frac{s^{\alpha} e_{1}(0)}{s^{\alpha}+4+3 e^{-s \tau}}=0 .
$$


At the same time, from (30), we can get

$$
\begin{aligned}
\lim _{t \rightarrow+\infty} e_{3}(t) & \\
\quad= & \lim _{s \rightarrow 0^{+}} s E_{3}(s) \\
\quad & \frac{s^{\alpha} e_{3}(0)+s\left(-8-4 e^{-s \tau}\right) E_{1}(s)}{s^{\alpha}+9+2 e^{-s \tau}}=0 \\
\lim _{t \rightarrow+\infty} e_{2}(t) & \\
= & \lim _{s \rightarrow 0^{+}} s E_{2}(s) \\
= & \frac{s^{\alpha} e_{2}(0)+s\left(-6-3 e^{-s \tau}\right) E_{1}(s)+s\left(-3-e^{-s \tau}\right) E_{3}(s)}{s^{\alpha}+7.5+5.3 e^{-s \tau}} \\
= & 0 .
\end{aligned}
$$

The above analysis implies that the fractional-order drive system (23) and the fractional-order response system (24) with the active control law (27) are synchronized.

4.1. Simulation Research. Here, let $a_{1}=35, b_{1}=3$, and $c_{1}=$ 28 in the Lü system and parameters of the Chen system are the same as in Section 3.1. The fractional order $\alpha$ is taken to be 0.78 and assume $\tau=1$. The initial conditions for drive and response system are $x_{1}(0)=0.2, y_{1}(0)=0$, $z_{1}(0)=0.5$, and $x_{2}(0)=-2, y_{2}(0)=2, z_{2}(0)=-4$, respectively. Thus initial conditions for the error system (28) are $e_{1}(0)=-2.2, e_{2}(0)=2$, and $e_{3}(0)=-4.5$. Figures 6(a)$6(\mathrm{c})$ show the synchronization between Chen and Lü system. The errors $e_{i}(t)$ for the drive and response system are shown in Figure 6(d).

\section{Chaos Synchronization between Fractional Order Time-Delay Lorenz and Chen System}

Assuming that Chen system is synchronized with Lorenz system, define the drive system as

$$
\begin{gathered}
\frac{d^{\alpha} x_{1}}{d t^{\alpha}}=a_{1}\left(y_{1}-x_{1}\right), \\
\frac{d^{\alpha} y_{1}}{d t^{\alpha}}=c_{1} x_{1}-y_{1}-x_{1} z_{1}, \\
\frac{d^{\alpha} z_{1}}{d t^{\alpha}}=x_{1} y_{1}-b_{1} z_{1}(t-\tau)
\end{gathered}
$$

and the Chen system as the response system

$$
\begin{gathered}
\frac{d^{\alpha} x_{2}}{d t^{\alpha}}=a_{2}\left(y_{2}-x_{2}\right)+u_{1}(t), \\
\frac{d^{\alpha} y_{2}}{d t^{\alpha}}=\left(c_{2}-a_{2}\right) x_{2}-x_{2} z_{2}+c_{2} y_{2}+u_{2}(t), \\
\frac{d^{\alpha} z_{2}}{d t^{\alpha}}=x_{2} y_{2}-b_{2} z_{2}(t-\tau)+u_{3}(t) .
\end{gathered}
$$

Let $e_{1}=x_{2}-x_{1}, e_{2}=y_{2}-y_{1}$, and $e_{3}=z_{2}-z_{1}$ be error functions. For synchronization it is essential that the errors $e_{i} \rightarrow 0$ as $t \rightarrow 0$. Then we can get

$$
\begin{gathered}
\frac{d^{\alpha} e_{1}}{d t^{\alpha}}=a_{2}\left(y_{2}-x_{2}\right)+u_{1}(t)-a_{1}\left(y_{1}-x_{1}\right), \\
\frac{d^{\alpha} e_{2}}{d t^{\alpha}}=\left(c_{2}-a_{2}\right) x_{2}-x_{2} z_{2}+c_{2} y_{2} \\
+u_{2}(t)-c_{1} y_{1}+x_{1} z_{1}+y_{1}, \\
\frac{d^{\alpha} e_{3}}{d t^{\alpha}}=x_{2} y_{2}-b_{2} z_{2}(t-\tau)+u_{3}(t)-x_{1} y_{1}+b_{1} z_{1}(t-\tau) .
\end{gathered}
$$

By using Matignon's theorem [44], so we here define active control function $u_{i}(i=1,2,3)$ as

$$
\begin{aligned}
u_{1}(t)= & -8 e_{1}-7 e_{1}(t-\tau)-6 e_{2}-5 e_{2}(t-\tau) \\
& -4 e_{3}-3 e_{3}(t-\tau)-a_{2}\left(y_{2}-x_{2}\right)+a_{1}\left(y_{1}-x_{1}\right) \\
u_{2}(t)= & -4.8 e_{2}-2.7 e_{1}(t-\tau)-2.9 e_{3}-1.3 e_{3}(t-\tau) \\
& -\left(c_{2}-a_{2}\right) x_{2}+x_{2} z_{2}-c_{2} y_{2}+c_{1} y_{1}-x_{1} z_{1}-y_{1} \\
u_{3}(t)= & -2 e_{3}-e_{3}(t-\tau)-x_{2} y_{2}+b_{2} z_{2}(t-\tau) \\
& +x_{1} y_{1}-b_{1} z_{1}(t-\tau)
\end{aligned}
$$

Substituting (36) into (35), we achieve

$$
\begin{gathered}
\frac{d^{\alpha} e_{1}}{d t^{\alpha}}=-8 e_{2}-7 e_{2}(t-\tau)-6 e_{2}-5 e_{2}(t-\tau) \\
-4 e_{3}-3 e_{3}(t-\tau), \\
\frac{d^{\alpha} e_{2}}{d t^{\alpha}}=-4.8 e_{2}-2.7 e_{2}(t-\tau)-2.9 e_{3}-1.3 e_{3}(t-\tau), \\
\frac{d^{\alpha} e_{3}}{d t^{\alpha}}=-2 e_{3}-e_{3}(t-\tau) .
\end{gathered}
$$

Then we start to prove that the error system (37) is asymptotically stable; that is, the system (33) and the system (34) with the active control law (36) are synchronized.

Taking the Laplace transformation in two sides of (37), letting $E_{i}(s)=L\left(e_{i}(t)\right)(i=1,2,3)$, and utilizing $L\left(d^{\alpha} e_{i} /\right.$ $\left.d t^{\alpha}\right)=s^{\alpha} E_{i}(s)-s^{\alpha-1} e_{i}(0)$ (see [18]), we obtain

$$
\begin{aligned}
s^{\alpha} E_{1}(s)-s^{\alpha-1} e_{1}(0)= & -8 E_{1}(s)-7 E_{1}(s) e^{-s \tau} \\
& -6 E_{2}(s)-5 E_{2}(s) e^{-s \tau} \\
& -4 E_{3}(s)-3 E_{3}(s) e^{-s \tau}, \\
s^{\alpha} E_{2}(s)-s^{\alpha-1} e_{2}(0)= & -4.8 E_{2}(s)-2.7 E_{2}(s) e^{-s \tau} \\
& -2.9 E_{3}(s)-1.3 E_{3}(s) e^{-s \tau}, \\
s^{\alpha} E_{3}(s)-s^{\alpha-1} e_{3}(0)=- & 2 E_{3}(s)-E_{3}(s) e^{-s \tau} .
\end{aligned}
$$




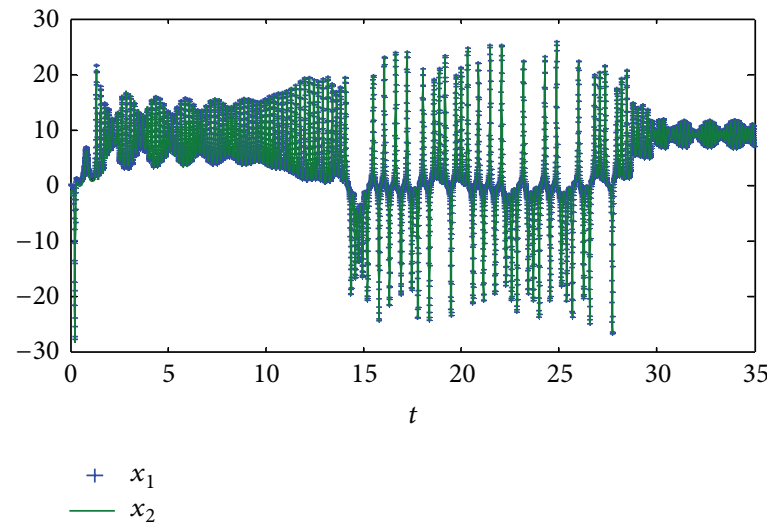

(a)

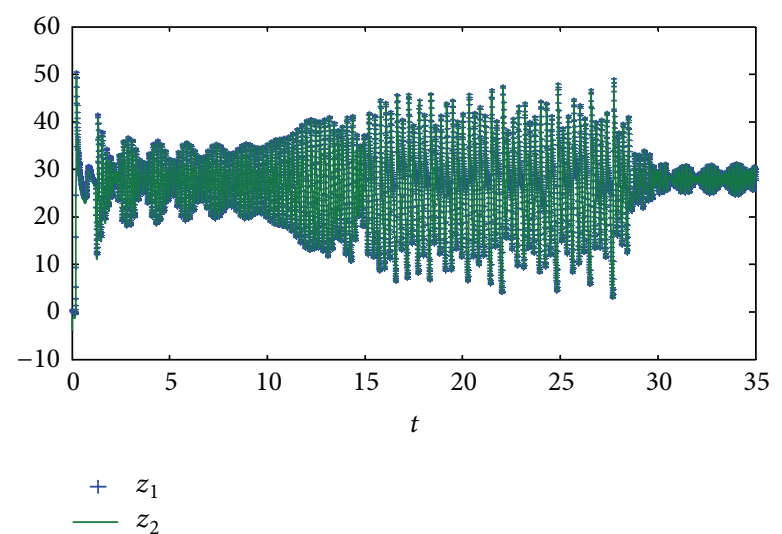

(c)

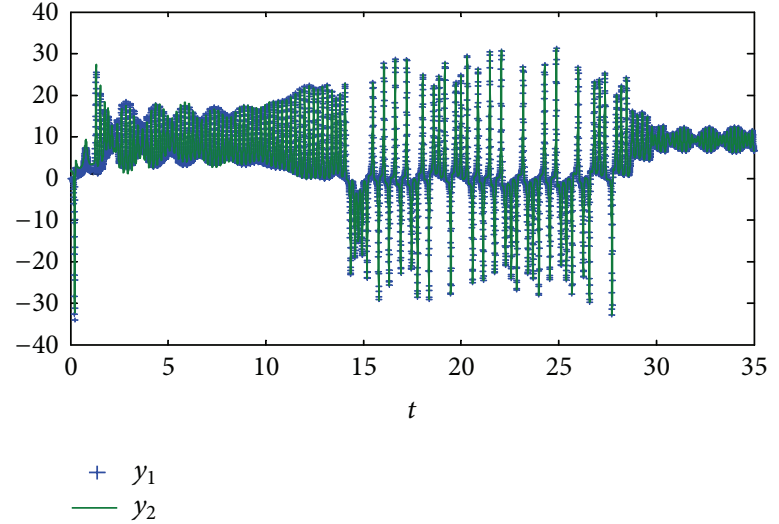

(b)

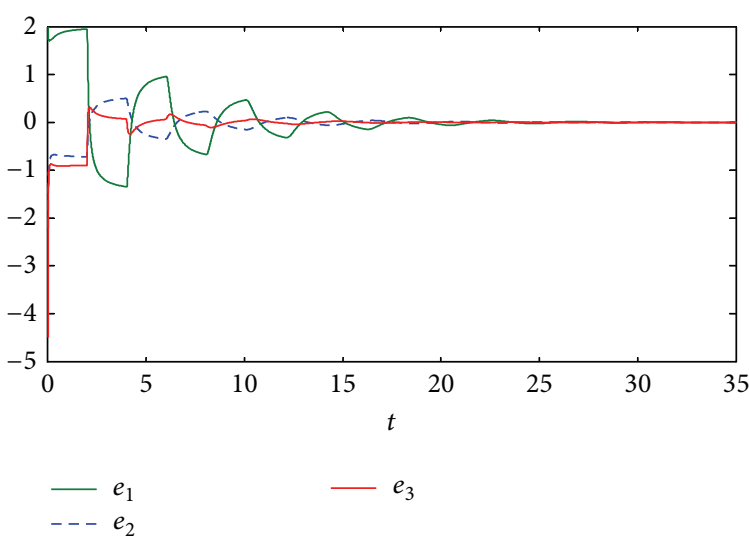

(d)

Figure 6: Chaos synchronization between (23) and (24). (a) Signals $x_{1}, x_{2}$. (b) Signals $y_{1}, y_{2}$. (c) Signals $z_{1}, z_{2}$. (d) Error system.

Equation (38) can be rewritten as follows:

$$
\begin{aligned}
& E_{1}(s) \\
& \quad=\frac{s^{\alpha-1} e_{1}(0)+\left(-6-5 e^{-s \tau}\right) E_{2}(s)+\left(-4-3 e^{-s \tau}\right) E_{3}(s)}{s^{\alpha}+8+7 e^{-s \tau}}, \\
& E_{2}(s)=\frac{s^{\alpha-1} e_{2}(0)+\left(-2.9-1.3 e^{-s \tau}\right) E_{3}(s)}{s^{\alpha}+4.8+2.7 e^{-s \tau}}, \\
& E_{3}(s)=\frac{s^{\alpha-1} e_{3}(0)}{s^{\alpha}+2+e^{-s \tau}} .
\end{aligned}
$$

By use of the final-value theorem of the Laplace transformation, we have

$$
\lim _{t \rightarrow+\infty} e_{3}(t)=\lim _{s \rightarrow 0^{+}} E_{3}(s)=\frac{s^{\alpha} e_{3}(0)}{s^{\alpha}+2+e^{-s \tau}}=0 .
$$

At the same time, from (39), we can get

$$
\begin{aligned}
\lim _{t \rightarrow+\infty} e_{2}(t) \\
\quad=\lim _{s \rightarrow 0^{+}} s E_{2}(s) \\
=\frac{s^{\alpha} e_{2}(0)+s\left(-2.9-1.3 e^{-s \tau}\right) E_{3}(s)}{s^{\alpha}+4.8+2.7 e^{-s \tau}}=0
\end{aligned}
$$

$$
\begin{aligned}
\lim _{t \rightarrow+\infty} & e_{1}(t) \\
& =\lim _{s \rightarrow 0^{+}} s E_{1}(s) \\
& =\frac{s^{\alpha} e_{1}(0)+s\left(-6-5 e^{-s \tau}\right) E_{2}(s)+s\left(-4-3 e^{-s \tau}\right) E_{3}(s)}{s^{\alpha}+8+7 e^{-s \tau}} \\
& =0 .
\end{aligned}
$$

So the error system (37) is asymptotically stable and chaos synchronization between the two systems is achieved.

5.1. Simulation Research. Here, we take parameters for fractional order time-delay Lorenz system as $a_{1}=10, b_{1}=8 / 3$, and $c_{1}=28$ and parameters of the Chen system are the same as in Section 3.1. The fractional order $\alpha$ is taken to be 0.95 and assume $\tau=0.4$. The initial conditions for the two systems are $x_{1}(0)=-8, y_{1}(0)=2, z_{1}(0)=3$, and $x_{2}(0)=4, y_{2}(0)=-5$, and $z_{2}(0)=6$, respectively. Hence the initial conditions for the error system $(37)$ are $e_{1}(0)=12, e_{2}(0)=-7$, and $e_{3}(0)=3$. Figures $7(\mathrm{a})-7(\mathrm{c})$ show the synchronization between Chen and Lorenz system. The errors $e_{i}(t)$ for the drive and response system are shown in Figure 7(d). 

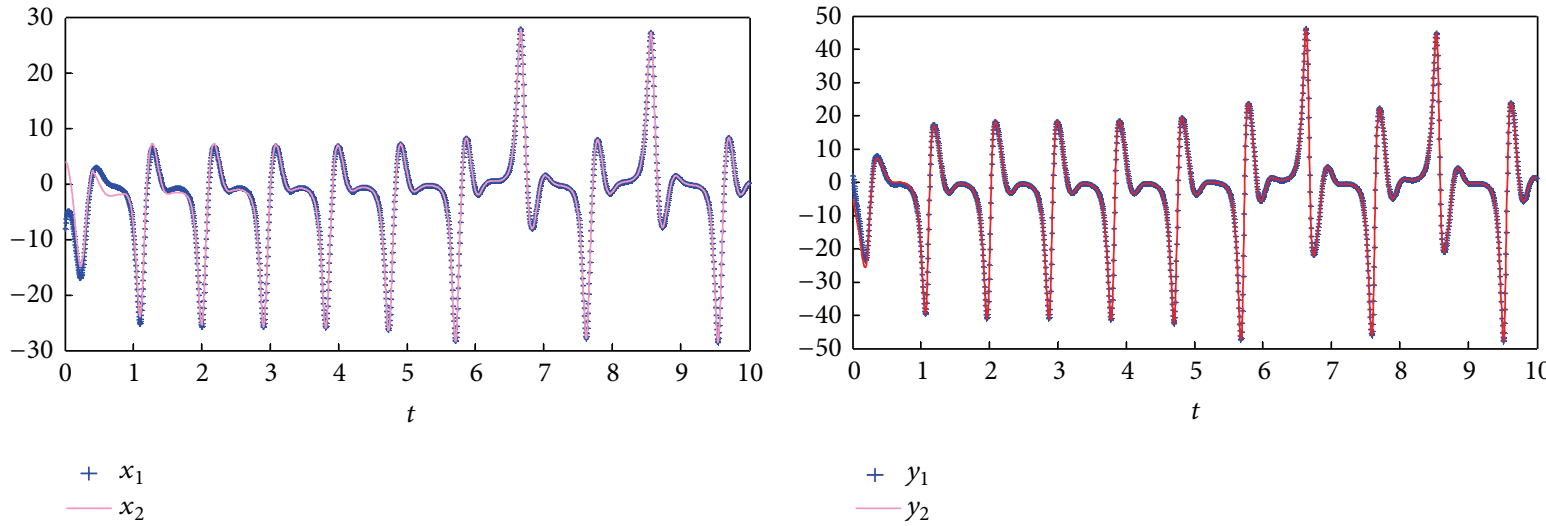

(a)
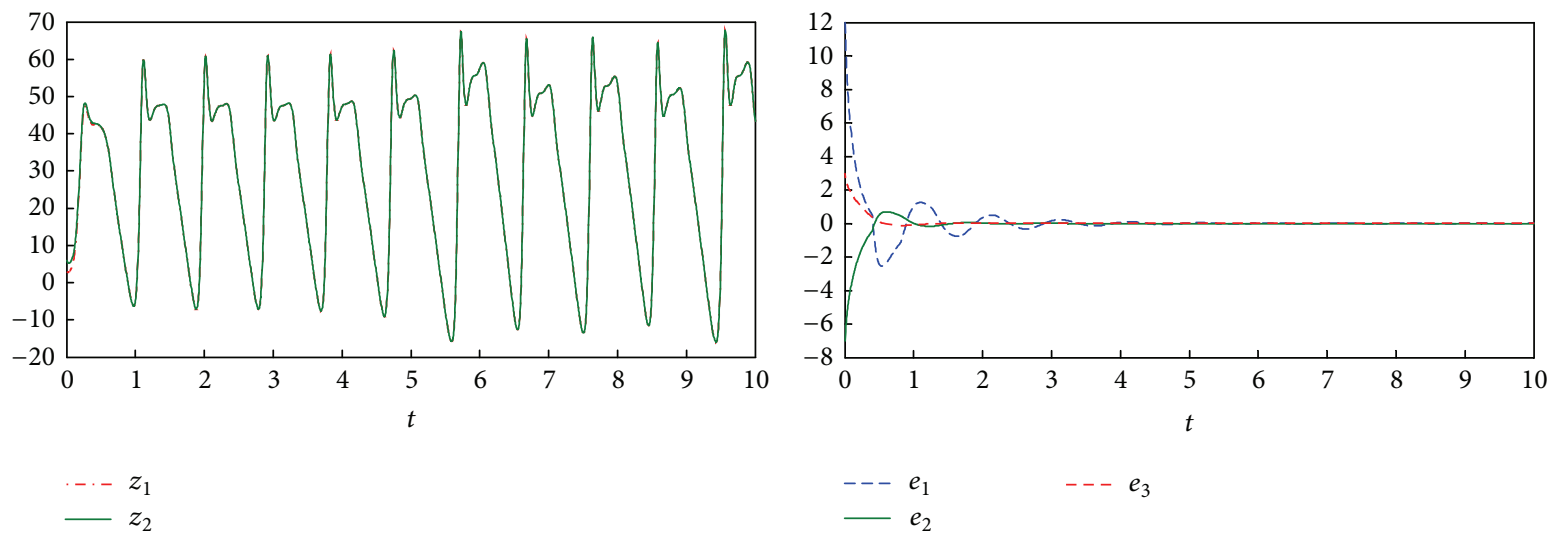

(c)

(d)

FIGURE 7: Chaos synchronization between (33) and (34). (a) Signals $x_{1}, x_{2}$. (b) Signals $y_{1}, y_{2}$. (c) Signals $z_{1}, z_{2}$. (d) Error system.

TABLE 1: Errors for different values of $\alpha$ and $t$.

\begin{tabular}{|c|c|c|c|c|c|c|}
\hline$t$ & $\alpha=0.985$ & $\alpha=0.92$ & $\alpha=0.86$ & $\alpha=0.79$ & $\alpha=0.75$ & $\alpha=0.72$ \\
\hline & \multicolumn{6}{|c|}{$e_{1}(t) 10^{-5}$} \\
\hline 5 & 14.810 & 53.589 & 64.378 & 60.895 & 55.085 & 49.929 \\
\hline 8 & 5.4515 & 20.460 & 25.487 & 25.197 & 23.386 & 21.610 \\
\hline \multirow[t]{2}{*}{10} & 3.4284 & 13.070 & 16.534 & 16.655 & 15.629 & 14.563 \\
\hline & \multicolumn{6}{|c|}{$e_{2}(t) 10^{-5}$} \\
\hline 5 & 1.4810 & 5.2970 & 6.3961 & 6.1012 & 5.5483 & 5.0483 \\
\hline 8 & 0.55838 & 2.0920 & 2.6062 & 2.5806 & 2.3980 & 2.2177 \\
\hline \multirow[t]{2}{*}{10} & 0.3352 & 1.3509 & 1.7069 & 1.7190 & 1.6131 & 1.5029 \\
\hline & \multicolumn{6}{|c|}{$e_{3}(t) 10^{-5}$} \\
\hline 5 & 3.0197 & 10.877 & 12.963 & 12.079 & 10.794 & 9.6725 \\
\hline 8 & 1.0866 & 4.0671 & 5.0380 & 4.9228 & 4.5231 & 4.1391 \\
\hline 10 & 0.67821 & 2.5795 & 3.2468 & 3.2359 & 3.0081 & 2.7774 \\
\hline
\end{tabular}

\section{Effects of Order $\alpha$ and Time Delay $\tau$ on Synchronization}

Recently, fractional order chaotic systems have been focus of attention. Generally speaking, there is a lowest order in a fractional order chaotic system. From the literature [23-25], we know that the order $\alpha$ affects the behavior of fractional chaotic dynamical systems. As shown in $[35,45]$, the time delay $\tau$ also affects the behavior of chaotic dynamical systems. In the section, we pay attention to the effects of the order $\alpha$ and the delay $\tau$ on synchronization. In Table 1 , some results are given about the error functions for different values of order in system (13) and system (14). Here experiments are done for fixed value of the delay $\tau=0.002$. It is obvious that the error in synchronization firstly increases and then decreases as the order $\alpha$ is decreased, which is not the case 
TABLE 2: Errors for different values of $\tau$ and $t$.

\begin{tabular}{|c|c|c|c|c|c|c|}
\hline$t$ & $\tau=0.1$ & $\tau=0.4$ & $\tau=0.8$ & $\tau=1$ & $\tau=1.5$ & $\tau=2$ \\
\hline & \multicolumn{6}{|c|}{$e_{1}(t) 10^{-6}$} \\
\hline 30 & 6.4058 & 8.1702 & 10.551 & 11.816 & 13.629 & 17.216 \\
\hline 32 & 5.5652 & 7.1822 & 9.3213 & 10.4366 & 13.0955 & 16.1873 \\
\hline \multirow[t]{2}{*}{35} & 4.8842 & 6.1556 & 8.0039 & 8.2546 & 10.0036 & 13.2583 \\
\hline & \multicolumn{6}{|c|}{$e_{2}(t) 10^{-6}$} \\
\hline 30 & 5.3471 & 10.9933 & 15.9716 & 71.8276 & 12800 & 18800 \\
\hline 32 & 4.7002 & 9.4500 & 78.9665 & 194.0404 & 63455 & 7420 \\
\hline \multirow[t]{2}{*}{35} & 3.9123 & 7.8744 & 15.0866 & 88.1245 & 4950 & 28500 \\
\hline & \multicolumn{6}{|c|}{$e_{3}(t) 10^{-6}$} \\
\hline 30 & 7.4512 & 5.4716 & 2.1277 & 0.5743 & 3.1265 & 7.0165 \\
\hline 32 & 6.5074 & 4.4264 & 1.7500 & 0.44107 & 2.9125 & 6.4450 \\
\hline 35 & 5.4538 & 3.7911 & 1.4449 & 0.3588 & 2.4521 & 5.3607 \\
\hline
\end{tabular}

in [46]. At the same time, we have summarized some results about the error functions for different values of the time delay $\tau$ in system (23) and system (24). In these observations, we take the order $\alpha=0.78$. From Table 2 , it is clear that the error functions $e_{1}(t)$ and $e_{2}(t)$ become bigger as the delay $\tau$ is increased. But the error function $e_{3}(t)$ firstly decreases and then increases.

\section{Conclusions}

In the paper, chaos synchronization of different fractional order time-delay chaotic systems is considered. Based on the Laplace transform theory, the conditions for achieving synchronization of different fractional order time-delay chaotic systems are analyzed by use of active control technique. Then numerical simulations are provided to verify the effectiveness and feasibility of the developed method. Finally, effects of the fraction order and the time delay on synchronization are further discussed.

\section{Conflict of Interests}

The authors declare that there is no conflict of interests regarding the publication of this paper.

\section{Acknowledgments}

This work was supported by the Natural Science Foundation of China (Grant Nos. 60872073, 51075068, and 60975017), by the Foundation of Huaqiao University (Grant Nos. 12BS228 and 13BS103), and by the Natural Science Foundation of Fujian Province (Grant No. 2013H2002). The authors would like to thank the reviewers for their valuable suggestions and comments.

\section{References}

[1] L. M. Pecora and T. L. Carroll, "Synchronization in chaotic systems," Physical Review Letters, vol. 64, no. 8, pp. 821-824, 1990.
[2] R. Aguilar-López and R. Martinez-Guerra, "Partial synchronization of different chaotic oscillators using robust PID feedback," Chaos, Solitons and Fractals, vol. 33, no. 2, pp. 572-581, 2007.

[3] G. Solís-Perales, V. Ayala, W. Kliemann, and R. Femat, "Complete synchronizability of chaotic systems: a geometric approach," Chaos, vol. 13, no. 2, pp. 495-501, 2003.

[4] X. F. Wang and G. R. Chen, "Synchronization in small-world dynamical networks," International Journal of Bifurcation and Chaos, vol. 12, no. 1, article 187, 2002.

[5] M. G. Rosenblum, A. S. Pikovsky, and J. Kurths, "Phase synchronization of chaotic oscillators," Physical Review Letters, vol. 76, no. 11, pp. 1804-1807, 1996.

[6] C. Sarasola, F. J. Torrealdea, A. d'Anjou, and M. Graña, "Cost of synchronizing different chaotic systems," Mathematics and Computers in Simulation, vol. 58, no. 4-6, pp. 309-327, 2002.

[7] G. Zhang, Z. Liu, and Z. Ma, "Generalized synchronization of different dimensional chaotic dynamical systems," Chaos, Solitons \& Fractals, vol. 32, no. 2, pp. 773-779, 2007.

[8] R. Brown, "Approximating the mapping between systems exhibiting generalized synchronization," Physical Review Letters, vol. 81, no. 22, pp. 4835-4838, 1998.

[9] R. Mainieri and J. Rehacek, "Projective synchronization in three-dimensional chaotic systems," Physical Review Letters, vol. 82, no. 15, pp. 3042-3045, 1999.

[10] C. P. Li, W. G. Sun, and D. L. Xu:, "Synchronization of complex dynamical networks with nonlinear inner-coupling functions and time delays," Progress of Theoretical Physics, vol. 114, no. 4, pp. 749-761, 2005.

[11] F. Chen and W. Zhang, "LMI criteria for robust chaos synchronization of a class of chaotic systems," Nonlinear Analysis, Theory, Methods and Applications, vol. 67, no. 12, pp. 3384-3393, 2007.

[12] J.-J. Yan, Y.-S. Yang, T.-Y. Chiang, and C.-Y. Chen, "Robust synchronization of unified chaotic systems via sliding mode control," Chaos, Solitons and Fractals, vol. 34, no. 3, pp. 947-954, 2007.

[13] Q. Chen, X. Ren, and J. Na, "Robust anti-synchronization of uncertain chaotic systems based on multiple-kernel least squares support vector machine modeling," Chaos, Solitons and Fractals, vol. 44, no. 12, pp. 1080-1088, 2011.

[14] J. K. Hale and S. M. V. Lunel, Introduction to Functional Differential Equations, Springer, New York, NY, USA, 1991. 
[15] W. Deng, Y. Wu, and C. Li, "Stability analysis of differential equations with time-dependent delay," International Journal of Bifurcation and Chaos, vol. 16, no. 2, pp. 465-472, 2006.

[16] C.-K. Cheng, H.-H. Kuo, Y.-Y. Hou, C.-C. Hwang, and T.-L. Liao, "Robust chaos synchronization of noise-perturbed chaotic systems with multiple time-delays," Physica A, vol. 387, no. 13, pp. 3093-3102, 2008.

[17] Y. Xiao, W. Xu, S. Tang, and X. Li, "Adaptive complete synchronization of the noise-perturbed two bi-directionally coupled chaotic systems with time-delay and unknown parametric mismatch," Applied Mathematics and Computation, vol. 213, no. 2, pp. 538-547, 2009.

[18] W. He, F. Qian, J. Cao, and Q.-L. Han, "Impulsive synchronization of two nonidentical chaotic systems with time-varying delay," Physics Letters A, vol. 375, no. 3, pp. 498-504, 2011.

[19] J.-P. Richard, "Time-delay systems: an overview of some recent advances and open problems," Automatica, vol. 39, no. 10, pp. 1667-1694, 2003.

[20] I. Podlubny, Fractional Differential Equations, vol. 198 of Mathematics in Science and Engineering, Academic Press, San Diego, Calif, USA, 1999.

[21] R. Hifer, Applications of Fractional Calculus in Physics, World Scientific, River Edge, NJ, USA, 2001.

[22] M. W. Hirsch and S. Smale, Differential Equations: Dynamical Systems and Linear Algebra, vol. 60, Academic Press, New York, NY, USA, 1974, Pure and Applied Mathematics.

[23] X. Wu, J. Li, and G. Chen, "Chaos in the fractional order unified system and its synchronization," Journal of the Franklin Institute, vol. 345 , no. 4, pp. 392-401, 2008.

[24] J. G. Lu and G. Chen, "A note on the fractional-order Chen system," Chaos, Solitons and Fractals, vol. 27, no. 3, pp. 685-688, 2006.

[25] J. G. Lu, "Chaotic dynamics of the fractional-order Ikeda delay system and its synchronization," Chinese Physics, vol. 15, no. 2, article 301, 2006.

[26] Y. Chen and K. L. Moore, "Analytical stability bound for delayed second-order systems with repeating poles using Lambert function W,' Automatica, vol. 38, no. 5, pp. 891-895, 2002.

[27] Y. Q. Chen and K. L. Moore, "Analytical stability bound for a class of delayed fractional-order dynamic systems," Nonlinear Dynamics, vol. 29, no. 1-4, pp. 191-200, 2002.

[28] W. H. Deng and C. P. Li, "Synchronization of chaotic fractional Chen system," Journal of the Physical Society of Japan, vol. 74, no. 6, pp. 1645-1648, 2005.

[29] W. H. Deng, C. P. Li, and J. H. Lü, "Stability analysis of linear fractional differential system with multiple time delays," Nonlinear Dynamics, vol. 48, no. 4, pp. 409-416, 2007.

[30] H. Zhu, S. Zhou, and Z. He, "Chaos synchronization of the fractional-order Chen's system," Chaos, Solitons and Fractals, vol. 41, no. 5, pp. 2733-2740, 2009.

[31] S. Q. Shao, X. Gao, and X. W. Liu, "Projective synchronization in coupled fractional order chaotic Rossler system and its control," Chinese Physics, vol. 16, no. 9, p. 2612, 2007.

[32] G. H. Erjaee and S. Momani, "Phase synchronization in fractional differential chaotic systems," Physics Letters A, vol. 372, no. 14, pp. 2350-2354, 2008.

[33] M. P. Lazarević and A. M. Spasić, "Finite-time stability analysis of fractional order time-delay systems: Gronwall's approach," Mathematical and Computer Modelling, vol. 49, no. 3-4, pp. 475481, 2009.
[34] H. Taghvafard and G. H. Erjaee, "Phase and anti-phase synchronization of fractional order chaotic systems via active control," Communications in Nonlinear Science and Numerical Simulation, vol. 16, no. 10, pp. 4079-4088, 2011.

[35] S. Bhalekar and V. Daftardar-Gejji, "Fractional ordered Liu system with time-delay," Communications in Nonlinear Science and Numerical Simulation, vol. 15, no. 8, pp. 2178-2191, 2010.

[36] I. Grigorenko and E. Grigorenko, "Chaotic dynamics of the fractional Lorenz system," Physical Review Letters, vol. 91, no. 3, pp. 034101/1-034101/4, 2003.

[37] X.-J. Wu and S.-L. Shen, "Chaos in the fractional-order Lorenz system," International Journal of Computer Mathematics, vol. 86, no. 7, pp. 1274-1282, 2009.

[38] G. Chen and T. Ueta, "Yet another chaotic attractor," International Journal of Bifurcation and Chaos, vol. 9, no. 7, pp. 14651466, 1999.

[39] C. Liu, L. Liu, and T. Liu, "A novel three-dimensional autonomous chaos system," Chaos, Solitons and Fractals, vol. 39, no. 4, pp. 1950-1958, 2009.

[40] V. Daftardar-Gejji and S. Bhalekar, "Chaos in fractional ordered Liu system," Computers \& Mathematics with Applications, vol. 59, no. 3, pp. 1117-1127, 2010.

[41] K. Diethelm, "An algorithm for the numerical solution of differential equations of fractional order," Electronic Transactions on Numerical Analysis, vol. 5, pp. 1-6, 1997.

[42] K. Diethelm and N. J. Ford, "Analysis of fractional differential equations," Journal of Mathematical Analysis and Applications, vol. 265, no. 2, pp. 229-248, 2002.

[43] K. Diethelm, N. J. Ford, and A. D. Freed, "A predictor-corrector approach for the numerical solution of fractional differential equations," Nonlinear Dynamics, vol. 29, no. 1-4, pp. 3-22, 2002.

[44] D. Matignon, "Stability results for fractional differential equations with applications to control processing," in Proceedings of the Computational Engineering in Systems and Application MultiConference, vol. 2, pp. 963-968, Lille, France, 1996.

[45] L. Li, H. Peng, Y. Yang, and X. Wang, "On the chaotic synchronization of Lorenz systems with time-varying lags," Chaos, Solitons and Fractals, vol. 41, no. 2, pp. 783-794, 2009.

[46] S. Bhalekar and V. Daftardar-Gejji, "Synchronization of different fractional order chaotic systems using active control," Communications in Nonlinear Science and Numerical Simulation, vol. 15, no. 11, pp. 3536-3546, 2010. 


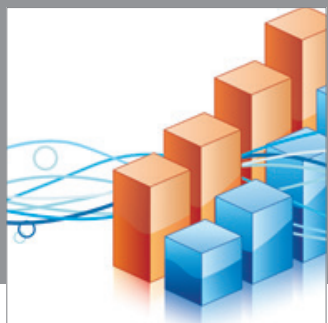

Advances in

Operations Research

mansans

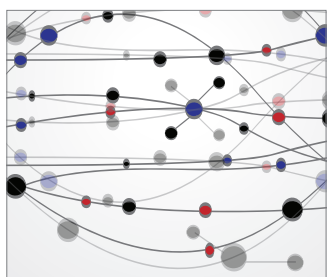

The Scientific World Journal
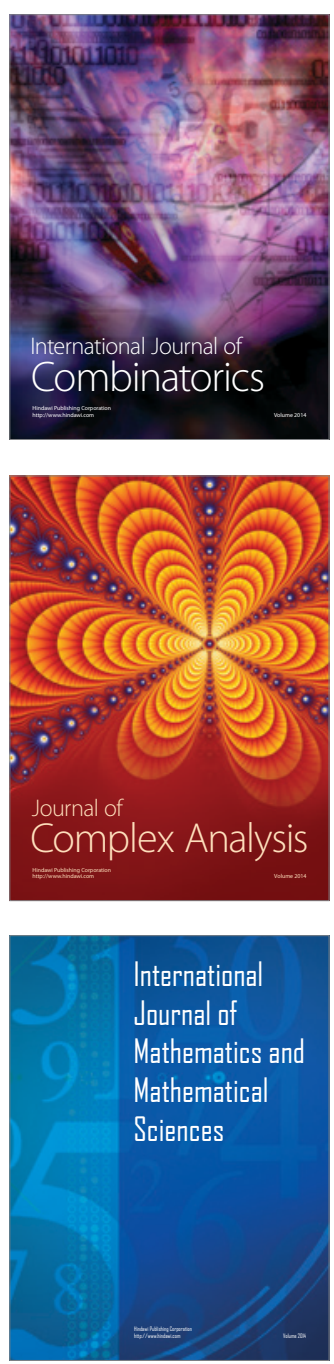
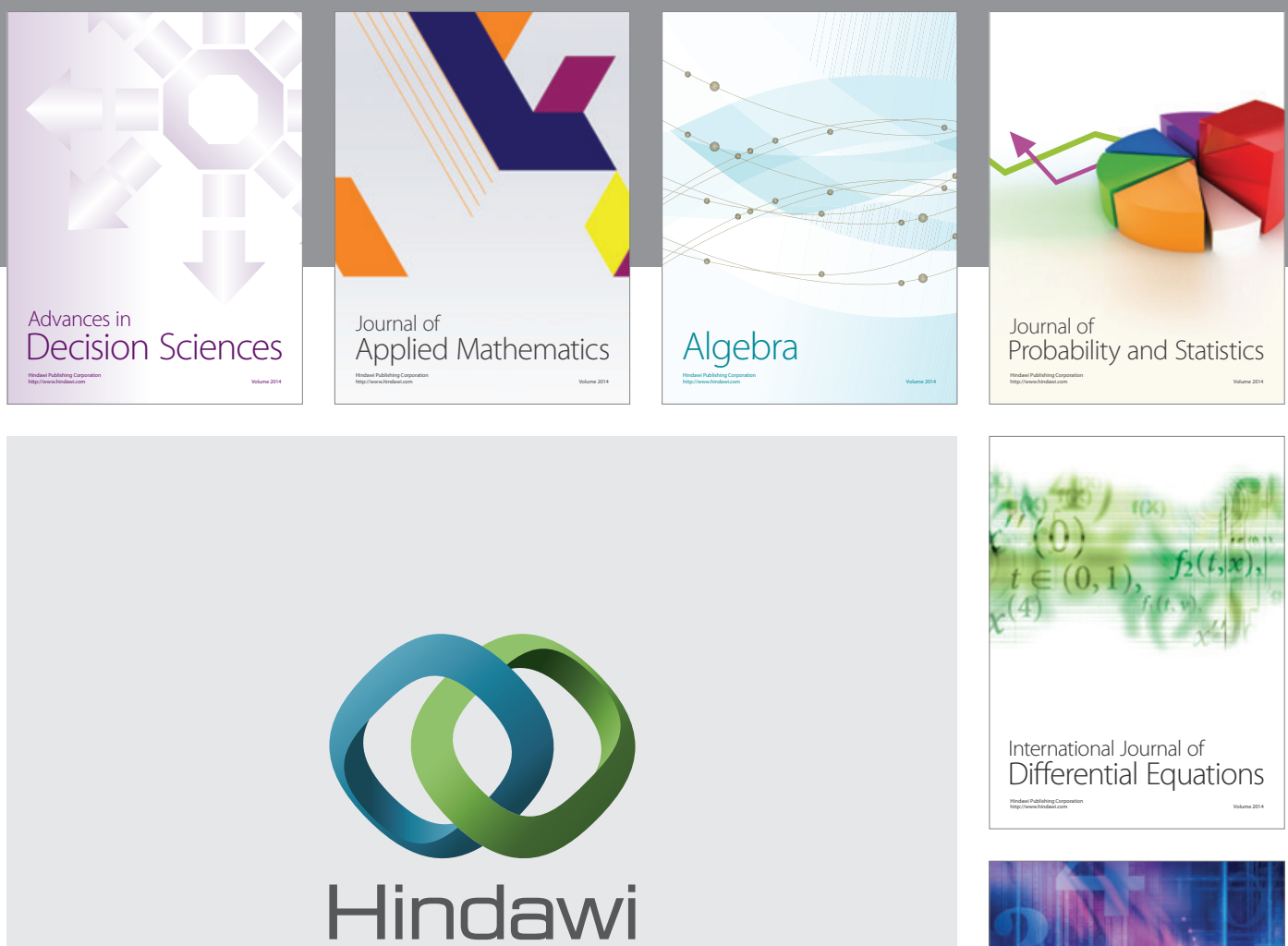

Submit your manuscripts at http://www.hindawi.com
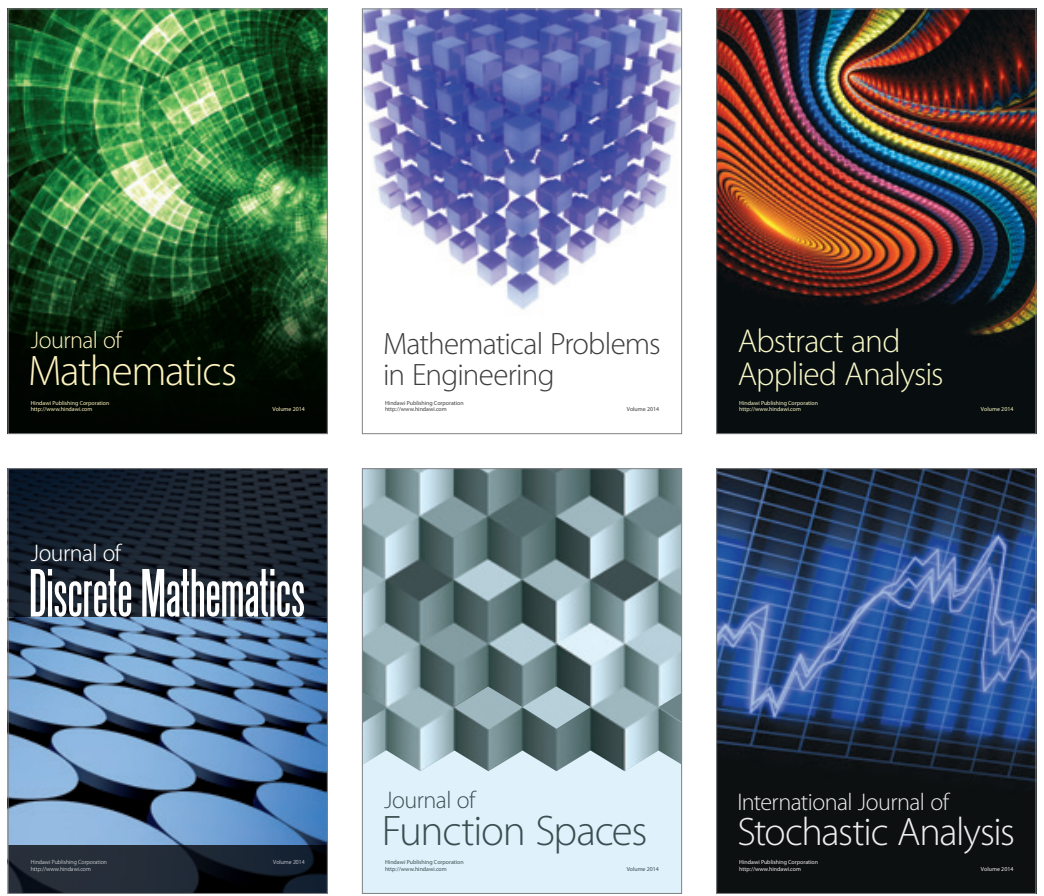

Journal of

Function Spaces

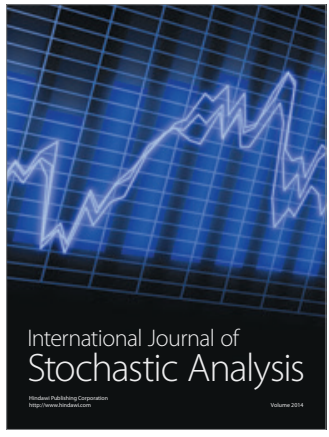

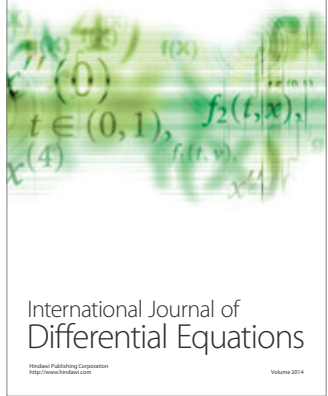
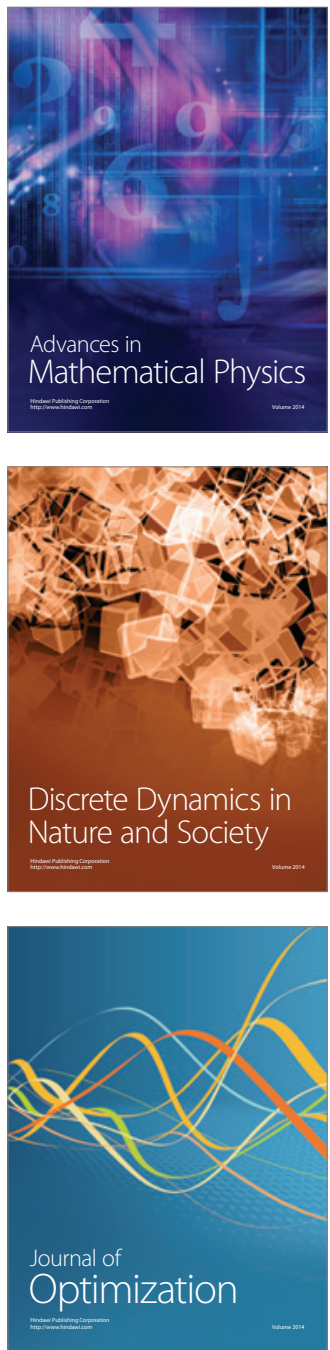